\title{
Analysis of Neurological Clinical Manifestations and Diagnostic Workups in Pediatric COVID-19: A Systematic Review
}

Gilbert Sterling Octavius ( $\square$ Sterlinggilbert613@hotmail.com )

Universitas Pelita Harapan Fakultas Kedokteran https://orcid.org/0000-0002-6439-6265

Shally Chandra

Universitas Pelita Harapan Fakultas Kedokteran

Jeremiah Hilkiah Wijaya

Universitas Pelita Harapan Fakultas Kedokteran

Andry Juliansen

Universitas Pelita Harapan Fakultas Kedokteran

\section{Research Article}

Keywords: COVID-19, SARS-CoV-2, Pediatric, Neurological, CSF Analysis

Posted Date: June 9th, 2021

DOI: https://doi.org/10.21203/rs.3.rs-535249/v1

License: () (1) This work is licensed under a Creative Commons Attribution 4.0 International License. Read Full License 


\section{Abstract}

Background While numerous studies report neurological manifestations of COVID-19, there are fewer reviews in the pediatric population, focusing solely on the manifestations.

Aim This systematic review aims to describe the clinical manifestations and laboratory findings of pediatric patients with neurological manifestations of COVID-19.

Methods We searched Pubmed, Science Direct, Medline, Scielo, Medrxiv, Research Square, SSRN, and Biorxiv with the appropriate keywords. Inclusion criteria include new-onset neurological manifestations with a time correlation to the confirmed COVID-19 with a further diagnostic work-up. We use The Joanna Briggs Institute's (JBI) essential evaluation checklist and Newcastle Ottawa Quality Assessment Scale (NOS) to evaluate the quality of the studies. The protocol for this systematic review has been registered in The international Prospective Register of Systematic Review (PROSPERO) database (CRD42021228671)

Results 31 articles were ultimately chosen with 42 patients (26 male) included in this systematic review. The neurological diagnosis was mostly of the CNS classification (38/42) with a predomination of brain disorders (22/38). Most patients had lymphopenia, elevated C-reactive protein, procalcitonin, ferritin, creatinine kinase, blood urea nitrogen, D-dimer, and erythrocyte sedimentation rate. In the CNS group, CSF monocytes (55\%; 11-80\%), CSF polymorphonuclear (PMN) cells (15\%; 0-96\%), CSF red blood cells (282 cells $/ \mathrm{mm}^{3} ; 2-4000$ cells $\left./ \mathrm{mm}^{3}\right)$, and opening pressure $\left(34.5 \mathrm{~cm} / \mathrm{H}_{2} \mathrm{O} ; 25-100 \mathrm{~cm} / \mathrm{H}_{2} \mathrm{O}\right)$ were elevated.

Conclusions Our systematic review found that neurological manifestations in COVID-19 occurred more often in males, with a majority being CNS disorders. A majority had a favorable outcome. More studies are needed in order to elucidate the viral mechanisms of SARS-CoV-2 in CSF.

\section{Introduction}

Over a year has passed since COVID-19 is declared a pandemic, and there are no signs of it slowing down $(1,2)$. Numerous strategies have been employed from social distancing, adequate personal protective equipment, closing down schools and other public services, and ramping up vaccine administrations (36). However, these measures have mixed results, with some countries are more successful than the others (7).

SARS-CoV-2 has undergone extensive research, and its impact ranges from asymptomatic to even death, with almost every aspect of physical and mental health affected $(2,8,9)$. Neurological manifestation in pediatric and adult populations is a known complication of COVID-19, and numerous studies are backing this finding $(10-12)$.

However, most of the neurological studies in COVID-19 are based on the adult population and mainly report neurological symptoms or complications (13-17). Similarly, when reviews are done in the pediatric population, these studies also focus heavily on the pathogenesis or clinical manifestations in the neurological department (18-20). Analysis of lumbar puncture, a functional diagnostic study that may help differentiate the etiology of infections in the nervous system, is also heavily done in the adult populations $(21-23)$.

Besides theories of pathogenesis and clinical manifestations or complications in neurological pediatric COVID-19 cases, there is still a lack of other clinical findings such as brain imaging, laboratory values, or cerebrospinal fluid (CSF) analysis, which may characterize pediatric patients with neurological manifestations in the setting of COVID-19 infection. While there are numerous case series, cohort studies, or even multi-center studies on this topic, the data are too heterogeneous, with different studies having different objectives of the studies (24-26).

Therefore, this systematic review aims to describe the clinical manifestations and outcomes, laboratory findings (such as routine blood work and inflammatory markers), radiology findings (such as brain computed tomography scan or brain magnetic resonance imaging), and specific neurological tests (such as electroencephalography and CSF analysis).

\section{Methods}

This systematic review adhered to the Preferred Reporting Items for Systematic Review and Meta-Analysis (PRISMA) 2020 statement (27). The protocol for this systematic review has been registered in The international Prospective Register of Systematic Review (PROSPERO) database (CRD42021228671).

The authors included case reports, case series, cross-sectional studies, and cohort studies that studied neurological impacts and their neurological diagnosis about pediatric COVID-19. Inclusion criteria included new-onset neurological manifestations with a time correlation to the diagnosis of confirmed COVID-19. The other inclusion criteria also ensured that the neurological manifestations must be investigated either by one of the following tests: laboratory testings, brain imaging, electroencephalogram (EEG), and lumbar puncture and CSF analysis. Exclusion criteria comprised co-infection by other pathogens confirmed by microbiological testings, neurological comorbidities before presentation, certain study types such as editorials and reviews, and multisystem inflammatory syndrome in children (MIS-C).

The literature search was limited from 2020 onwards with no restrictions on language. In order to ensure literature selection, references from reviews and all studies included in this systematic review were scanned and included if they fulfilled the inclusion criteria.

The literature search started on January 14, 2021, and ended on January 15, 2021. The authors used four unique databases, such as PubMed, Science Direct, Medline, and Scielo, and four different preprint databases such as Medrxiv, Research Square, Social Science Research Network (SSRN), and Biorxiv. The keywords are listed in Table 1. Each research's data was compiled in a standardized format, including study citations, demographic characteristics of the 
included participants, neurological manifestations and diagnosis, COVID-19 severity, patient's length of stay and outcomes, laboratory and imaging results, as well as CSF analysis. The corresponding author of that particular study will be sent an email if some data are missing pertaining to this systematic review.

Table 1

Sets of keywords used for this systematic review in each database

\begin{tabular}{|ll|}
\hline Database & Keywords \\
\hline $\begin{array}{l}\text { First set } \\
\text { second }\end{array}$ & COVID-19; COVID-2019; severe acute respiratory syndrome coronavirus 2; 2019-nCoV; SARS-CoV-2; 2019 novel coronavirus; 2019 nCoV \\
\hline Third set & $\begin{array}{l}\text { cerebrospinal fluid; liquor cerebrospinalis; lumbar puncture; lumbal puncture; computed tomography scan; magnetic resonance imaging; } \\
\text { electroencephalography; EEG }\end{array}$ \\
\hline $\begin{array}{l}\text { Fourth } \\
\text { set }\end{array}$ & $\begin{array}{l}\text { neurological; neurology; central nervous system; CNS; brain; manifestation; symptom; presentation; feature; complication; peripheral nervous } \\
\text { system; neuropathy; skeletal muscle; myositis; neuromuscular junction; headache; anosmia; olfactory; ageusia; cranial neuropathy; cranial } \\
\text { nerve; seizures; encephalitis; meningitis; stroke; cerebrovascular disease; cerebral hemorrhage; intracerebral hemorrhage; cerebral infarct; } \\
\text { cortical venous thrombosis; deep cerebral venous thrombosis; impaired consciousness; confusion; weakness; Guillain-Barre syndrome; Miller } \\
\text { Fisher syndrome; ataxia; myopathy; myelitis; myelopathy; encephalopathy; meningoencephalitis; convulsion; neuropsychiatric }\end{array}$ \\
\hline
\end{tabular}

Dong et al. classified COVID-19 severity as follows: (a) asymptomatic (absence of signs and symptoms associated with COVID-19, routine clinical imaging, but positive ribonucleic acid SARS-CoV-2 test); (b) mild (presence of symptoms limited to upper respiratory tract infections, such as fever, fatigue, myalgia, cough, sore throat, runny nose or nasal congestion, or gastrointestinal symptoms); (c) moderate (presence of mild symptoms plus clinical signs and symptoms of pneumonia but no hypoxemia); (d) severe (presence of mild symptoms plus dyspnea, central cyanosis, and oxygen saturation of 92 percent); and (e) critical (presence of mild symptoms plus clinical signs and symptoms of pneumonia but no hypoxemia) (presence of acute respiratory distress syndrome, respiratory failure, encephalopathy, myocardial injury, coagulation dysfunction, and acute kidney injury) (28).

Three independent reviewers (GS, SC, JH) conducted the initial search and the quality assessment of each study. The Joanna Briggs Institute's (JBI) essential evaluation checklist for case reports was used to measure the general consistency of case series and case reports (29). In contrast, the Newcastle Ottawa Quality Assessment Scale was used to assess case management and longitudinal studies (NOS) (30). Any differences between JBI and NOS results were discussed until a conclusion could be reached. If there were still any unresolved disagreements, an expert reviewer (AJ) was consulted, and the decision was made based on his expertise and consensus. In order to be included in this systematic review, the case reports needed to fulfill the majority of JBI criteria and scored $\geq 7$ in NOS score.

Age and gender, the severity of COVID-19 events, fatality, laboratory values, and variations in CSF protein and cell count markers dependent on the central nervous system (CNS) or peripheral nervous system (PNS) classification were all assessed using pooled descriptive tests.

\section{Results}

There were 2073 articles found initially, and 31 articles were ultimately chosen for this systematic review (Fig. 1). Almost all of the articles are case reports or case series except four studies (31-34). The list and specifications of each study are listed in Table 2 . There are 42 patients included in this systematic review, with 26 of them being male. When EEG was done, it was primarily due to acute symptomatic seizure or concomitant encephalopathy, and all the results $(n=$ 11 ) showed some abnormalities. There are 11 computed tomography (CT) scans (6/11 abnormal findings), 20 magnetic resonance imaging (MRI) (14/20 abnormal findings), and two magnetic resonance angiography (MRA) (2/2 abnormal findings) done for the brain or spinal cord. Imaging was done in infectious diseases such as encephalitis to rule out underlying pathologies, vascular diseases such as stroke and aneursym to locate the exact anatomical location, and other miscellaneous diseases such as Guillain-Barré Syndrome (GBS) and transverse myelitis. 
Table 2

Study details

\begin{tabular}{|c|c|c|c|c|c|c|c|c|c|}
\hline$\#$ & $\begin{array}{l}\text { First author } \\
\text { (year) }\end{array}$ & $\begin{array}{l}\text { No. of } \\
\text { patients } \\
(\% \\
\text { male) }\end{array}$ & $\begin{array}{l}\text { Age } \\
\text { (years)* }\end{array}$ & $\begin{array}{l}\text { Neurological } \\
\text { signs and } \\
\text { symptoms }(\mathrm{N})\end{array}$ & $\begin{array}{l}\text { Neurological } \\
\text { diagnosis }(\mathrm{N})\end{array}$ & $\begin{array}{l}\text { Brain imaging } \\
\text { results }(N)\end{array}$ & EEG results $(\mathrm{N})$ & $\begin{array}{l}\text { COVID-19 } \\
\text { severity }(\mathrm{N})\end{array}$ & Treatm€ \\
\hline (31) & $\begin{array}{l}\text { Miller, E } \\
(2020)\end{array}$ & $2(100)$ & $\begin{array}{l}0.795 \\
(0.02- \\
11)\end{array}$ & $\begin{array}{l}\text { Non-specific: } \\
\text { Fever (2), } \\
\text { generalized } \\
\text { weakness (1) }\end{array}$ & $\begin{array}{l}\text { Status epilepticus } \\
\text { (1), acute } \\
\text { symptomatic } \\
\text { seizure (1) }\end{array}$ & N/A & $\begin{array}{l}\text { Near continuous } \\
\text { sharply } \\
\text { contoured } \\
\text { slowing with rare } \\
\text { embedded sharp } \\
\text { waves, maximal } \\
\text { right parietal } \\
\text { region, frequent } \\
\text { runs of the non- } \\
\text { evolving rhythmic } \\
\text { delta of varying } \\
\text { amplitudes, } \\
\text { anterior (at times } \\
\text { right frontal or bi- } \\
\text { frontal } \\
\text { predominance) or } \\
\text { anterior/posterior, } \\
\text { excess overriding } \\
\text { beta activity, mild } \\
\text { to moderate } \\
\text { encephalopathy } \\
\text { with the absence } \\
\text { of posterior } \\
\text { dominant rhythm } \\
\text { (in one patient) }\end{array}$ & Mild (2) & N/A \\
\hline (32) & $\begin{array}{l}\text { Patterson, } \\
\mathrm{R}(2020)\end{array}$ & $1(0)$ & 16 & $\begin{array}{l}\text { Non-specific: } \\
\text { Headache, fever } \\
\text { PNS: Bilateral } \\
\text { failure of } \\
\text { abduction } \\
\text { CNS: Diplopia, } \\
\text { weakness of } \\
\text { left orbicularis } \\
\text { oculi }\end{array}$ & $\begin{array}{l}\text { Pseudotumor } \\
\text { cerebri (1) }\end{array}$ & $\begin{array}{l}\text { MRI: } \\
\text { dilated optic nerve } \\
\text { sheaths and } \\
\text { narrowed but } \\
\text { patent transverse } \\
\text { sinuses; } \\
\text { consistent with } \\
\text { raised intracranial } \\
\text { pressure. No } \\
\text { parenchymal } \\
\text { changes or } \\
\text { thrombosis of the } \\
\text { head \& neck } \\
\text { vessels. }\end{array}$ & $\mathrm{N} / \mathrm{A}$ & Moderate (1) & $\begin{array}{l}\text { Therapє } \\
\text { punctur } \\
\text { acetazo } \\
\text { colchici } \\
\text { myocarı }\end{array}$ \\
\hline (33) & $\begin{array}{l}\text { Emami, A } \\
(2020)\end{array}$ & $2(50)$ & 2.9 & $\begin{array}{l}\text { Non-specific: } \\
\text { Fever (2) } \\
\text { PNS: Bilateral } \\
\text { upward plantar } \\
\text { reflexes (1) } \\
\text { CNS: Seizure } \\
\text { (2), altered } \\
\text { mental status } \\
\text { (2) }\end{array}$ & $\begin{array}{l}\text { Status epilepticus } \\
\text { (1), tonic-clonic } \\
\text { seizure (1) }\end{array}$ & $\begin{array}{l}\text { CT scan: } \\
\text { right occipital } \\
\text { mass and } \\
\text { intracerebral } \\
\text { hemorrhage (1); } \\
\text { MRI: } \\
\text { right occipital } \\
\text { mass (suspected } \\
\text { ganglioglioma) } \\
\text { and intracerebral } \\
\text { hemorrhage }\end{array}$ & $\begin{array}{l}\text { Generalized } \\
\text { slowing (in one } \\
\text { patient) }\end{array}$ & $\begin{array}{l}\text { Mild (1), } \\
\text { Critical (1) }\end{array}$ & $\begin{array}{l}\text { Phenob } \\
\text { levetirac } \\
\text { valproa1 } \\
\text { phenyto } \\
\text { midazol } \\
\text { thiopent } \\
\text { fentany }\end{array}$ \\
\hline
\end{tabular}

*Data are presented in median (range) when there is more than one patient

${ }^{+}$Both investigations are done on the same patient

EEG, electroencephalogram; CSF, cerebrospinal fluid; LOS, length of stay; MRA, magnetic resonance angiography; MRI, magnetic resonance imaging; CT, com। posterior reversible encephalopathy syndrome; N/A, not available 


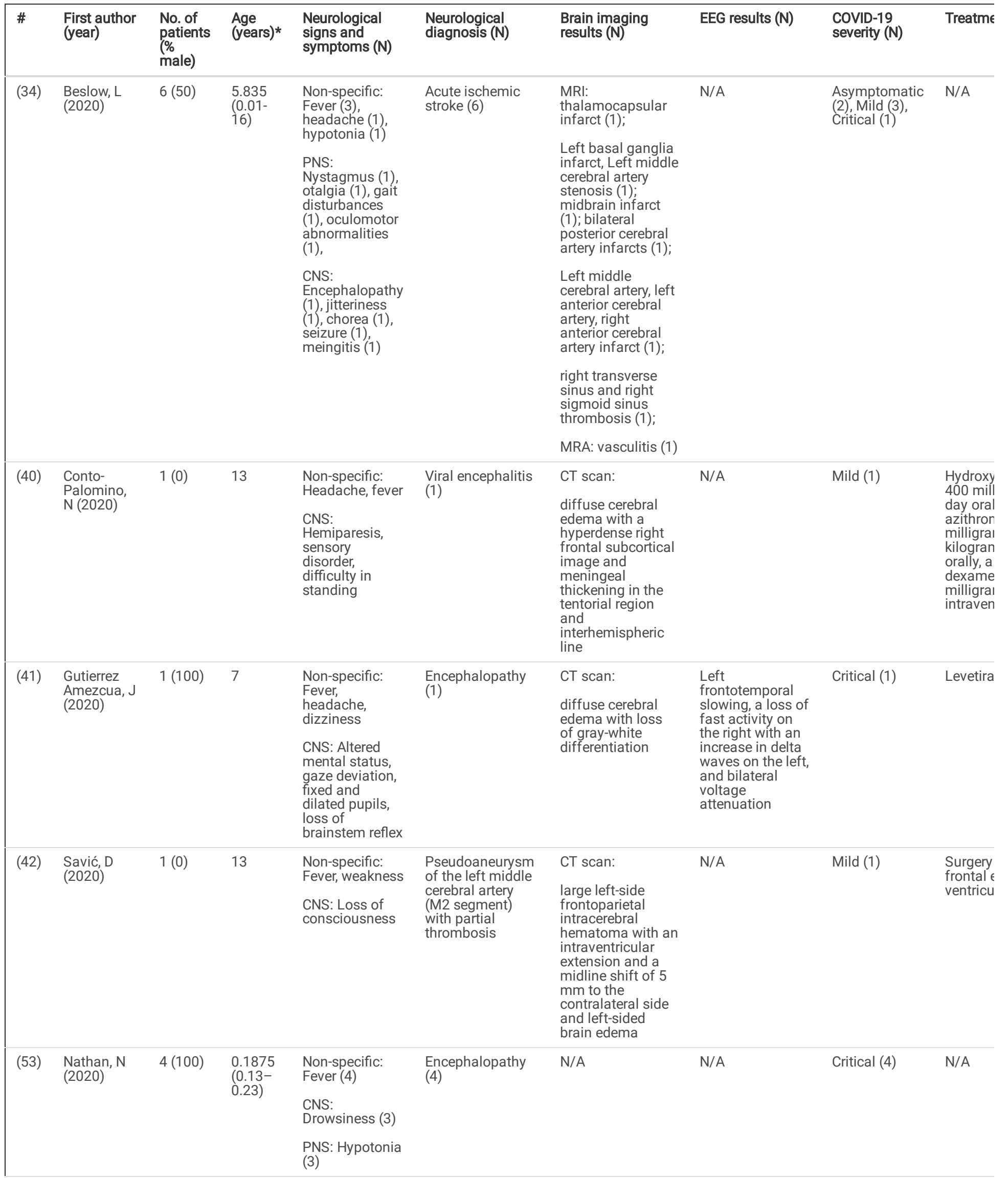

*Data are presented in median (range) when there is more than one patient

${ }^{+}$Both investigations are done on the same patient

EEG, electroencephalogram; CSF, cerebrospinal fluid; LOS, length of stay; MRA, magnetic resonance angiography; MRI, magnetic resonance imaging; CT, com। posterior reversible encephalopathy syndrome; N/A, not available 


\begin{tabular}{|c|c|c|c|c|c|c|c|c|c|}
\hline$\#$ & $\begin{array}{l}\text { First author } \\
\text { (year) }\end{array}$ & $\begin{array}{l}\text { No. of } \\
\text { patients } \\
\text { (\% } \\
\text { male) }\end{array}$ & $\begin{array}{l}\text { Age } \\
\text { (years)* }\end{array}$ & $\begin{array}{l}\text { Neurological } \\
\text { signs and } \\
\text { symptoms }(\mathrm{N})\end{array}$ & $\begin{array}{l}\text { Neurological } \\
\text { diagnosis }(\mathrm{N})\end{array}$ & $\begin{array}{l}\text { Brain imaging } \\
\text { results }(\mathrm{N})\end{array}$ & EEG results $(\mathrm{N})$ & $\begin{array}{l}\text { COVID-19 } \\
\text { severity }(\mathrm{N})\end{array}$ & Treatme \\
\hline (54) & $\begin{array}{l}\text { McAbee, G } \\
(2020)\end{array}$ & $1(100)$ & 11 & $\begin{array}{l}\text { Non-specific: } \\
\text { Generalized } \\
\text { weakness }\end{array}$ & $\begin{array}{l}\text { Viral encephalitis } \\
\text { (1) }\end{array}$ & CT scan: normal & $\begin{array}{l}\text { Frontal } \\
\text { Intermittent delta } \\
\text { activity }\end{array}$ & Mild (1) & N/A \\
\hline (55) & $\begin{array}{l}\text { Burr, }{ }^{\top} \\
(2021)\end{array}$ & $1(0)$ & 1.92 & $\begin{array}{l}\text { Non-specific: } \\
\text { Fever } \\
\text { CNS: Altered } \\
\text { mental status, } \\
\text { seizure, } \\
\text { hyperkinetic } \\
\text { movements }\end{array}$ & $\begin{array}{l}\text { Autoimmune } \\
\text { encephalitis (1) }\end{array}$ & MRI: normal & N/A & Mild (1) & $\begin{array}{l}\text { IV } \\
\text { metyhlp } \\
(30 \mathrm{mg} / \\
\text { IVIG }(2 \text { c } \\
\text { adminis } \\
\text { for the } 1 \\
\text { enceph' }\end{array}$ \\
\hline (56) & $\begin{array}{l}\text { Chacón- } \\
\text { Aguilar, R } \\
(2020)\end{array}$ & $1(100)$ & 0.07 & $\begin{array}{l}\text { Non-specific: } \\
\text { Lethargy } \\
\text { PNS: } \\
\text { Hypotonia, } \\
\text { areflexia } \\
\text { CNS: Altered } \\
\text { mental status }\end{array}$ & $\begin{array}{l}\text { Acute } \\
\text { symptomatic } \\
\text { seizure (1) }\end{array}$ & $\mathrm{N} / \mathrm{A}$ & $\begin{array}{l}\text { Continuous } \\
\text { patters with } \\
\text { sleep-wake } \\
\text { cycles }\end{array}$ & Mild (1) & $\begin{array}{l}\text { Empirici } \\
\text { antibiot }\end{array}$ \\
\hline (57) & $\begin{array}{l}\text { Di Nicola, P } \\
(2020)\end{array}$ & $1(0)$ & 0.049 & $\begin{array}{l}\text { Non-specific: } \\
\text { Fever, } \\
\text { generalized } \\
\text { weakness } \\
\text { CNS: Seizure }\end{array}$ & $\begin{array}{l}\text { Encephalopathy } \\
\text { (1) }\end{array}$ & MRI: normal & $\begin{array}{l}\text { Cortical } \\
\text { depression } \\
\text { without seizures }\end{array}$ & Critical (1) & $\begin{array}{l}\text { Broad-s } \\
\text { empirici } \\
\text { antimicl } \\
\text { caffein€ }\end{array}$ \\
\hline (58) & $\begin{array}{l}\text { Chegondi, } \\
\text { M (2020) }\end{array}$ & $1(0)$ & 2 & $\begin{array}{l}\text { Non-specific: } \\
\text { Fever } \\
\text { CNS: } \\
\text { Convulsion }\end{array}$ & $\begin{array}{l}\text { Status epilepticus } \\
\text { (1) }\end{array}$ & MRI: Normal & $\begin{array}{l}\text { Generalized } \\
\text { slowing without } \\
\text { recurrence of } \\
\text { seizures }\end{array}$ & Mild (1) & $\begin{array}{l}\text { IV lorazı } \\
\text { leveritac } \\
\text { acetami } \\
\text { Ceftriax } \\
\mathrm{mg} / \mathrm{kg} \text {, } \\
\text { fospher }\end{array}$ \\
\hline (59) & $\begin{array}{l}\text { Paybast, S } \\
(2020)\end{array}$ & $1(0)$ & 14 & $\begin{array}{l}\text { PNS: } \\
\text { Hyporeflexia, } \\
\text { lower limb } \\
\text { weakness, } \\
\text { hypoesthesia } \\
\text { CNS: } \\
\text { Quadriparesis, } \\
\text { ataxia }\end{array}$ & $\begin{array}{l}\text { Guillain-Barré } \\
\text { Syndrome (1) }\end{array}$ & $\mathrm{N} / \mathrm{A}$ & N/A & $\begin{array}{l}\text { Asymptomatic } \\
\text { (1) }\end{array}$ & $\begin{array}{l}\text { IVIG } 20 \\
\text { for five । }\end{array}$ \\
\hline$(60)$ & $\begin{array}{l}\text { Khalifa, M } \\
(2020)\end{array}$ & $1(100)$ & 11 & $\begin{array}{l}\text { Non-specific: } \\
\text { Fever, } \\
\text { hypotonia } \\
\text { PNS: Areflexia, } \\
\text { symmetrical } \\
\text { lower limb } \\
\text { weakness, } \\
\text { hypoesthesia, } \\
\text { gait } \\
\text { disturbances }\end{array}$ & $\begin{array}{l}\text { Guillain-Barré } \\
\text { Syndrome (1) }\end{array}$ & $\begin{array}{l}\text { MRI: } \\
\text { enhancement of } \\
\text { cauda equina }\end{array}$ & $\mathrm{N} / \mathrm{A}$ & Moderate (1) & $\begin{array}{l}\text { IVIG } 1 \mathrm{~g} \\
2 \text { days, } \\
\text { acetami } \\
\text { hydroxy } \\
\text { low mol } \\
\text { weight } \mathrm{F}\end{array}$ \\
\hline (61) & $\begin{array}{l}\text { Frank, C } \\
(2020)\end{array}$ & $1(100)$ & 15 & $\begin{array}{l}\text { Non-specific: } \\
\text { Fever, } \\
\text { headache, retro- } \\
\text { orbital pain, } \\
\text { hyperhidrosis } \\
\text { PNS: Areflexia, } \\
\text { symmetrical } \\
\text { lower limb } \\
\text { weakness }\end{array}$ & $\begin{array}{l}\text { Guillain-Barré } \\
\text { Syndrome (1) }\end{array}$ & MRI: normal & $\mathrm{N} / \mathrm{A}$ & Mild (1) & $\begin{array}{l}\text { Methylp } \\
\text { azithyro } \\
\text { albenda } \\
400 \mathrm{mg} \\
5 \text { days }\end{array}$ \\
\hline (62) & $\begin{array}{l}\text { Shen, M } \\
(2021)\end{array}$ & $1(100)$ & 1.42 & $\begin{array}{l}\text { CNS: Right } \\
\text { hemiparesis }\end{array}$ & $\begin{array}{l}\text { Acute ischemic } \\
\text { stroke (1) }\end{array}$ & $\begin{array}{l}\text { MRI: } \\
\text { acute left pontine } \\
\text { infarct }\end{array}$ & $\mathrm{N} / \mathrm{A}$ & $\begin{array}{l}\text { Asymptomatic } \\
\text { (1) }\end{array}$ & $\begin{array}{l}\text { Aspirin * } \\
\text { daily }\end{array}$ \\
\hline
\end{tabular}

*Data are presented in median (range) when there is more than one patient

${ }^{+}$Both investigations are done on the same patient

EEG, electroencephalogram; CSF, cerebrospinal fluid; LOS, length of stay; MRA, magnetic resonance angiography; MRI, magnetic resonance imaging; CT, com। posterior reversible encephalopathy syndrome; N/A, not available 


\begin{tabular}{|c|c|c|c|c|c|c|c|c|c|}
\hline$\#$ & $\begin{array}{l}\text { First author } \\
\text { (year) }\end{array}$ & $\begin{array}{l}\text { No. of } \\
\text { patients } \\
(\% \\
\text { male) }\end{array}$ & $\begin{array}{l}\text { Age } \\
\text { (years)* }\end{array}$ & $\begin{array}{l}\text { Neurological } \\
\text { signs and } \\
\text { symptoms }(\mathrm{N})\end{array}$ & $\begin{array}{l}\text { Neurological } \\
\text { diagnosis }(\mathrm{N})\end{array}$ & $\begin{array}{l}\text { Brain imaging } \\
\text { results }(\mathrm{N})\end{array}$ & EEG results $(\mathrm{N})$ & $\begin{array}{l}\text { COVID-19 } \\
\text { severity }(\mathrm{N})\end{array}$ & Treatme \\
\hline (63) & $\begin{array}{l}\text { de Ruijter, N } \\
(2020)\end{array}$ & $1(100)$ & 15 & $\begin{array}{l}\text { Non-specific: } \\
\text { Headache, } \\
\text { nausea, } \\
\text { photopsias } \\
\text { PNS: Visual } \\
\text { loss }\end{array}$ & $\begin{array}{l}\text { Neuromyelitis } \\
\text { optica spectrum } \\
\text { disorder (1) }\end{array}$ & $\begin{array}{l}\text { MRI: } \\
\text { Normal brain } \\
\text { appearance; Orbit: } \\
\\
\text { a bilateral } \\
\text { edematous optic } \\
\text { nerve lesion (right } \\
\text { more than left), } \\
\text { characteristic for } \\
\text { bilateral optic } \\
\text { neuritis }\end{array}$ & $\mathrm{N} / \mathrm{A}$ & Mild (1) & $\begin{array}{l}\text { Intraven } \\
\text { methylp } \\
\text { (1 g/day } \\
\text { days) }\end{array}$ \\
\hline (64) & $\begin{array}{l}\text { Dugue, } \mathrm{R} \\
(2020)\end{array}$ & $1(100)$ & 0.115 & $\begin{array}{l}\text { Non-specific: } \\
\text { Fever } \\
\text { CNS: Seizure, } \\
\text { gaze deviation } \\
\text { (sustained } \\
\text { upward gaze) }\end{array}$ & $\begin{array}{l}\text { Acute } \\
\text { symptomatic } \\
\text { seizure (1) }\end{array}$ & N/A & $\begin{array}{l}\text { An excess of } \\
\text { sharp temporal } \\
\text { transients for age } \\
\text { and intermittent } \\
\text { vertex delta } \\
\text { slowing with } \\
\text { normal sleep- } \\
\text { wake cycling }\end{array}$ & Mild (1) & N/A \\
\hline (65) & $\begin{array}{l}\text { Khan, A } \\
(2021)\end{array}$ & $1(100)$ & 11 & $\begin{array}{l}\text { Non-specific: } \\
\text { Fever } \\
\text { PNS: Visual } \\
\text { loss }\end{array}$ & $\begin{array}{l}\text { Myelin } \\
\text { oligodendrocyte } \\
\text { glycoprotein } \\
\text { (MOG) antibody } \\
\text { disease }\end{array}$ & $\begin{array}{l}\text { MRI: } \\
\text { bilateral } \\
\text { asymmetrical } \\
\text { optic neuritis, right } \\
\text { > left, involving the } \\
\text { intraorbital and } \\
\text { intracanalicular } \\
\text { portion on the } \\
\text { right side and } \\
\text { intraorbital portion } \\
\text { on the left side; } \\
\text { enhancement of } \\
\text { the optic nerve } \\
\text { sheath in the right } \\
\text { orbit; chiasmatic, } \\
\text { retrochiasmatic } \\
\text { portion, as well as } \\
\text { optic tracts, were } \\
\text { normal in signal } \\
\text { intensity and } \\
\text { appearance on } \\
\text { both sides }\end{array}$ & $\mathrm{N} / \mathrm{A}$ & Mild (1) & $\begin{array}{l}\text { Intraven } \\
\text { methylp } \\
\text { adminis } \\
30 \mathrm{mg} / \mathrm{k} \\
\text { by oral } \\
\text { prednis } \\
\mathrm{mg} / \mathrm{kg} / \mathrm{l}\end{array}$ \\
\hline
\end{tabular}

*Data are presented in median (range) when there is more than one patient

${ }^{+}$Both investigations are done on the same patient

EEG, electroencephalogram; CSF, cerebrospinal fluid; LOS, length of stay; MRA, magnetic resonance angiography; MRI, magnetic resonance imaging; CT, com। posterior reversible encephalopathy syndrome; N/A, not available 


\begin{tabular}{|c|c|c|c|c|c|c|c|c|c|}
\hline \# & $\begin{array}{l}\text { First author } \\
\text { (year) }\end{array}$ & $\begin{array}{l}\text { No. of } \\
\text { patients } \\
(\% \\
\text { male) }\end{array}$ & $\begin{array}{l}\text { Age } \\
\text { (years)* }\end{array}$ & $\begin{array}{l}\text { Neurological } \\
\text { signs and } \\
\text { symptoms }(\mathrm{N})\end{array}$ & $\begin{array}{l}\text { Neurological } \\
\text { diagnosis }(\mathrm{N})\end{array}$ & $\begin{array}{l}\text { Brain imaging } \\
\text { results }(N)\end{array}$ & EEG results $(\mathrm{N})$ & $\begin{array}{l}\text { CoVID-19 } \\
\text { severity }(\mathrm{N})\end{array}$ & Treatme \\
\hline (66) & $\begin{array}{l}\text { Dominguez, } \\
\text { J (2020) }\end{array}$ & $2(100)$ & $\begin{array}{l}8.5(4- \\
13)\end{array}$ & $\begin{array}{l}\text { Non-specific: } \\
\text { Fever (1), } \\
\text { headache (1), } \\
\text { weakness (1) } \\
\text { CNS: Loss of } \\
\text { sensory } \\
\text { disorder (1), } \\
\text { drowsy (1), } \\
\text { gaze deviation } \\
\text { (1), absent } \\
\text { patellar reflex } \\
\text { (1), flaccid } \\
\text { paralysis (1), } \\
\text { strabismus (1) }\end{array}$ & $\begin{array}{l}\text { Acute } \\
\text { disseminated } \\
\text { encephalomyelitis } \\
\text { (1), Guillain-Barré } \\
\text { Syndrome (1) }\end{array}$ & $\begin{array}{l}\text { CT scan: normal } \\
(1) ; \\
\text { multifocal, } \\
\text { hyperintense white } \\
\text { matter lesions in } \\
\text { the frontal region } \\
\text { right temporal. }\end{array}$ & $\mathrm{N} / \mathrm{A}$ & $\begin{array}{l}\text { Asymptomatic } \\
(1), \text { Mild (1) }\end{array}$ & $\begin{array}{l}\text { Ceftriax } \\
\text { milligraı } \\
\text { acyclov } \\
\text { milligraı } \\
\text { mannitc } \\
\text { grams/ } \\
\text { pulses c } \\
\text { methylp } \\
30 \\
\text { milligra। } \\
\text { for } 5 \text { da } \\
\text { Immunc } \\
400 \text { gra } \\
\text { for } 5 \text { da }\end{array}$ \\
\hline$(67)$ & $\begin{array}{l}\text { Seth, V } \\
(2020)\end{array}$ & $1(100)$ & 15 & $\begin{array}{l}\text { Non-specific: } \\
\text { Headache } \\
\text { CNS: } \\
\text { Photophobia, } \\
\text { irritable, } \\
\text { agitated }\end{array}$ & Encephalitis (1) & $\begin{array}{l}\text { CT scan: normal; } \\
\text { MRl: normal }\end{array}$ & $\mathrm{N} / \mathrm{A}$ & Mild (1) & $\begin{array}{l}\text { Intraven } \\
\text { acyclov } \\
\text { intraven } \\
\text { and par }\end{array}$ \\
\hline (68) & $\begin{array}{l}\text { Mirzaee, S } \\
(2020)\end{array}$ & $1(100)$ & 12 & $\begin{array}{l}\text { Non-specific: } \\
\text { Headache } \\
\text { CNS: Seizure, } \\
\text { dysarthria }\end{array}$ & $\begin{array}{l}\text { Focal cerebral } \\
\text { arteriopathy (1) }\end{array}$ & $\begin{array}{l}\text { MRI: } \\
\text { acute infarction } \\
\text { without } \\
\text { microhemorrhages } \\
\text { along with focal } \\
\text { irregular narrowing } \\
\text { and banding of } \\
\text { the proximal M1 } \\
\text { segment of the left } \\
\text { middle cerebral } \\
\text { artery with a } \\
\text { slightly reduced } \\
\text { distal flow }\end{array}$ & $\mathrm{N} / \mathrm{A}$ & Mild (1) & Diazepa \\
\hline (69) & $\begin{array}{l}\text { Kaur, H } \\
(2020)\end{array}$ & $1(0)$ & 3 & $\begin{array}{l}\text { Non-specific: } \\
\text { Weakness } \\
\text { CNS: Decreased } \\
\text { sensation, } \\
\text { hemiparesis, } \\
\text { absent gag } \\
\text { reflex, } \\
\text { quadriparesis, } \\
\text { areflexia, no } \\
\text { pain response }\end{array}$ & $\begin{array}{l}\text { Transverse } \\
\text { myelitis }\end{array}$ & $\begin{array}{l}\text { MRI: } \\
\text { swelling of the } \\
\text { cervical spinal } \\
\text { cord with T2- } \\
\text { hyperintense } \\
\text { edema on the } \\
\text { transverse aspect } \\
\text { of the spinal cord, } \\
\text { extending from the } \\
\text { lower medulla to } \\
\text { the midthoracic } \\
\text { level }\end{array}$ & $\mathrm{N} / \mathrm{A}$ & $\begin{array}{l}\text { Asymptomatic } \\
\text { (1) }\end{array}$ & $\begin{array}{l}\text { Intraven } \\
\text { methylp } \\
\text { ( } 30 \mathrm{mg} / \\
\text { and } \\
\text { immunc } \\
\mathrm{g} / \mathrm{kg} \text { tot } \\
\text { plasma } \\
(1.0-1.5 \\
\text { volumes } \\
\text { per sess } \\
\text { rituxima } \\
\text { infusion } \\
\left.\mathrm{mg} / \mathrm{m}^{2}\right)\end{array}$ \\
\hline (70) & $\begin{array}{l}\text { Bhatta, S } \\
(2020)\end{array}$ & $1(100)$ & 11 & $\begin{array}{l}\text { CNS: Seizure } \\
\text { (stretching and } \\
\text { tightening of all } \\
\text { four limbs, } \\
\text { uprolling of } \\
\text { eyes, frothing } \\
\text { from the mouth, } \\
\text { teeth clench, } \\
\text { and tongue bite } \\
\text { without urinary } \\
\text { or bowel } \\
\text { incontinence) }\end{array}$ & $\begin{array}{l}\text { Tonic-clonic } \\
\text { seizure (1) }\end{array}$ & CT scan: normal & N/A & $\begin{array}{l}\text { Asymptomatic } \\
(1)\end{array}$ & $\begin{array}{l}\text { Lorazep } \\
\text { Levetira }\end{array}$ \\
\hline (71) & $\begin{array}{l}\text { Swarz, J } \\
(2020)\end{array}$ & $1(100)$ & $\begin{array}{l}4.5 \\
(0.01-9)\end{array}$ & $\begin{array}{l}\text { Non-specific: } \\
\text { Fever } \\
\text { CNS: Decreased } \\
\text { consciousness }\end{array}$ & $\begin{array}{l}\text { Encephalopathy } \\
\text { and status } \\
\text { epilepticus (1) }\end{array}$ & $\begin{array}{l}\text { CT scan: normal; } \\
\text { MRl: normal }\end{array}$ & $\begin{array}{l}\text { Continuous delta } \\
\text { slowing } \\
\text { throughout the } \\
\text { right hemisphere } \\
\text { without } \\
\text { epileptiform } \\
\text { features }\end{array}$ & Critical (1) & $\begin{array}{l}\text { Lorazep } \\
\text { antibiot }\end{array}$ \\
\hline
\end{tabular}

*Data are presented in median (range) when there is more than one patient

${ }^{+}$Both investigations are done on the same patient

EEG, electroencephalogram; CSF, cerebrospinal fluid; LOS, length of stay; MRA, magnetic resonance angiography; MRI, magnetic resonance imaging; CT, com। posterior reversible encephalopathy syndrome; N/A, not available 


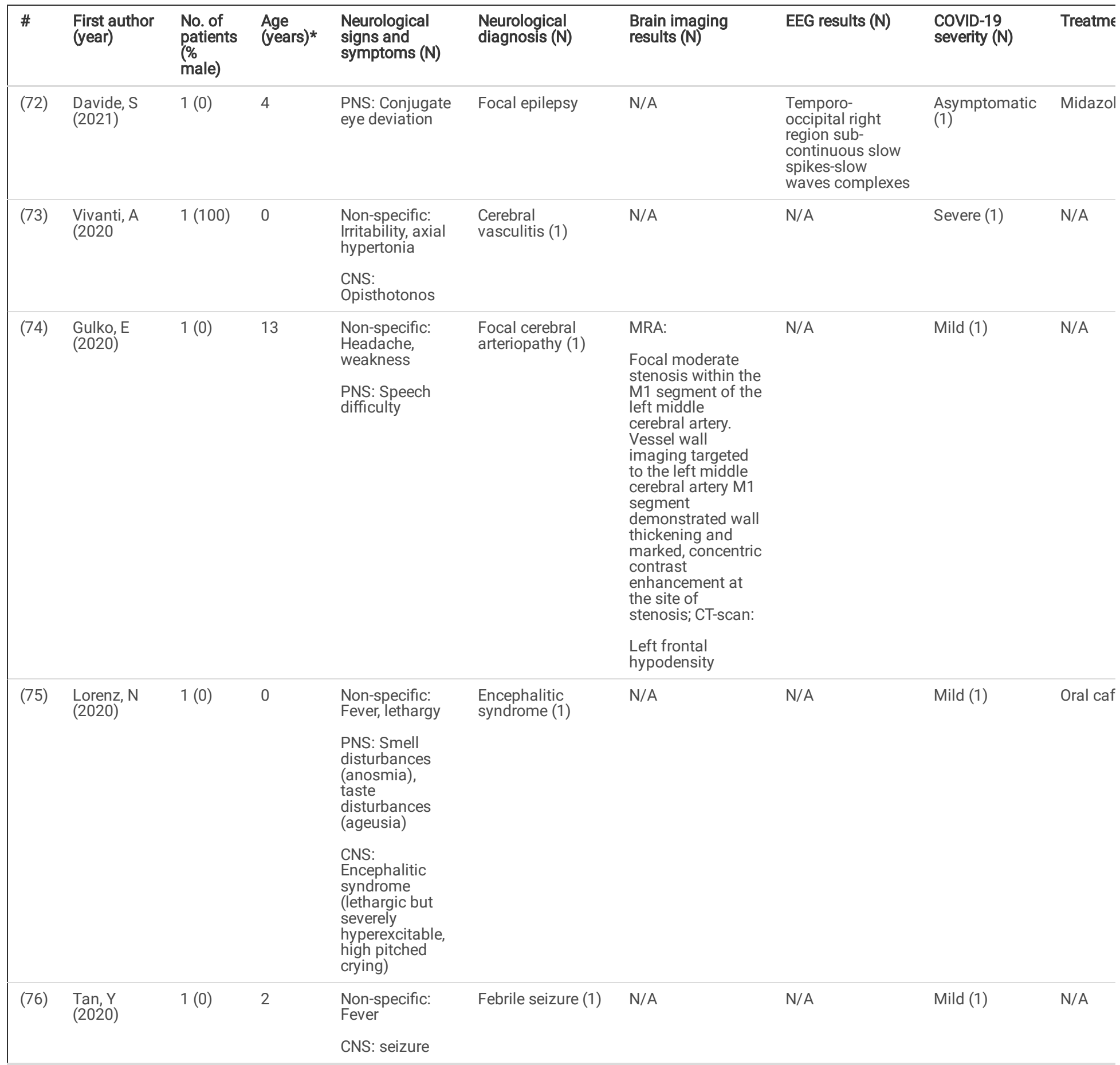

*Data are presented in median (range) when there is more than one patient

${ }^{+}$Both investigations are done on the same patient

EEG, electroencephalogram; CSF, cerebrospinal fluid; LOS, length of stay; MRA, magnetic resonance angiography; MRI, magnetic resonance imaging; CT, com। posterior reversible encephalopathy syndrome; N/A, not available

According to CNS or PNS manifestations, the neurological diagnosis was mainly of the CNS side (38/42) (Fig. 2). Brain disorders predominated in this category $(22 / 38)$, with the most common diagnosis being acute symptomatic seizure $(n=6)$ and encephalomyelopathy $(n=6)$. However, the single most common neurological diagnosis belonged to the vascular disorders (11/38), namely stroke, with seven patients diagnosed with COVID-19. In the PNS classification, all patients were affected by GBS (4/4).

The most common COVID-19 specific sign and symptom was fever $(n=29)$, followed by cough $(n=9)$, and vomiting $(n=8)$ (Fig. 3). As for COVID-19 neurological signs and symptoms, seizure and altered consciousness were the most common signs and symptoms, with nine cases each followed by headache $(n=8)$ and irritability and hemiparesis $(n=7)$ (Fig. 4). Out of the 18 PCR testing done from the CSF sample, five were positive for COVID-19 while two out of three rectal samples were positive for COVID-19 (Table 3). The median age of all the patients was 3.5 years old (0-16 years old). Out of 37 patients 
with known outcomes, 34 patients were alive (91.9\%). Length of stay was known in 31 patients and six days (0-30 days) was the median length of stay. Patients mostly presented with moderate COVID-19 severity (24/42), followed by asymptomatic (8/42) and mild presentation (7/42).

Table 3

Individualized patient characteristics

\begin{tabular}{|c|c|c|c|}
\hline Variables & \# Patients with available data & Median (Range) & $N(\%)$ \\
\hline Age (years) & 42 & $3.5(0-16)$ & $\mathrm{N} / \mathrm{A}$ \\
\hline Male gender & 42 & $\mathrm{~N} / \mathrm{A}$ & $26(61.9)$ \\
\hline CNS diagnosis & 42 & $\mathrm{~N} / \mathrm{A}$ & $37(88.1)$ \\
\hline First onset of symptom before first neurologic manifestation (days) & 18 & $2.5(0-10)$ & $\mathrm{N} / \mathrm{A}$ \\
\hline \multicolumn{4}{|l|}{ COVID-19 tests } \\
\hline Serologies & 9 & N/A & \\
\hline IgM positive & & & $0(0)$ \\
\hline IgG positive & & & $3(33.3)$ \\
\hline $\lg M$ and IgG positive & & & $2(22.2)$ \\
\hline Not specified* & & & $4(44.4)$ \\
\hline \multicolumn{4}{|l|}{ Polymerase chain reaction (PCR) } \\
\hline Positive from the cerebrospinal fluid sample & 18 & $\mathrm{~N} / \mathrm{A}$ & $5(27.8)$ \\
\hline Positive from nasopharyngeal sample & 35 & $\mathrm{~N} / \mathrm{A}$ & $33(94.3)$ \\
\hline Positive from rectal sample & 3 & $\mathrm{~N} / \mathrm{A}$ & $2(67.7)$ \\
\hline Outcome (alive) & 37 & $\mathrm{~N} / \mathrm{A}$ & 34 (91.9) \\
\hline Length of stay (days) & 31 & $6(0-30)$ & $\mathrm{N} / \mathrm{A}$ \\
\hline COVID-19 severity & 42 & & \\
\hline Asymptomatic & & $\mathrm{N} / \mathrm{A}$ & $8(19.0)$ \\
\hline Mild & & N/A & $5(11.9)$ \\
\hline Moderate & & $\mathrm{N} / \mathrm{A}$ & $24(57.1)$ \\
\hline Severe & & $\mathrm{N} / \mathrm{A}$ & $2(4.8)$ \\
\hline Critical & & $\mathrm{N} / \mathrm{A}$ & $3(7.1)$ \\
\hline \multicolumn{4}{|l|}{ *Presented as serologies positive } \\
\hline CNS, central nervous system; N/A, not available & & & \\
\hline
\end{tabular}

Table 4 presented a pooled analysis of laboratory values. Pediatric COVID-19 patients with neurological manifestations had lymphopenia, elevated C-reactive protein (CRP), procalcitonin, ferritin, creatinine kinase, blood urea nitrogen, D-dimer, and erythrocyte sedimentation rate (ESR). 
Table 4

Laboratory values

\begin{tabular}{|c|c|c|c|}
\hline Variable & \# Patients with available data & Reference range & Median (range) \\
\hline \multicolumn{4}{|l|}{ Hematology } \\
\hline Hemoglobin $(\mathrm{g} / \mathrm{dL})$ & 14 & $11.5-14.5$ & $11.75(7.2-15.0)$ \\
\hline White blood cell count $\left(10^{3} / \mu \mathrm{l}\right)$ & 18 & $4-12$ & $7.745(2.0-20.6)$ \\
\hline Lymphocyte (\%) & 8 & $25-33$ & $22.65(11.6-49.4)$ \\
\hline Neutrophil (\%) & 6 & $57-67$ & $57.7(22-83.7)$ \\
\hline Platelets $\left(10^{3} / \mu \mathrm{l}\right)$ & 12 & $150-400$ & $340.5(81-406)$ \\
\hline \multicolumn{4}{|l|}{ Inflammatory markers } \\
\hline C-reactive protein (mg/L) & 24 & Male $0.6-7.9$ Female $0.5-10.0$ & $7.55(0-556)$ \\
\hline Ferritin $(\mathrm{ng} / \mathrm{mL})$ & 5 & $10-60$ & $87.3(19.9-4220)$ \\
\hline Procalcitonin (ng/mL) & 7 & $\leq 0.15$ & $0.21(0.1-1.31)$ \\
\hline Lactate dehydrogenase (U/L) & 5 & $150-500$ & $390(128-1019)$ \\
\hline Creatine kinase $(\mathrm{U} / \mathrm{L})$ & 5 & $5-130$ & $246.8(43.7-482)$ \\
\hline Lactate (mg/dL) & 3 & $7-14$ & $5(4.32-7.0)$ \\
\hline \multicolumn{4}{|l|}{ Liver and kidney functions } \\
\hline Creatinine $(\mathrm{mg} / \mathrm{dL})$ & 3 & $0.3-0.7$ & $0.5(0.47-0.5)$ \\
\hline Alanine transaminase $(\mathrm{U} / \mathrm{L})$ & 5 & $5-45$ & $14(8-20)$ \\
\hline Aspartate aminotransferase (U/L) & 5 & $15-50$ & $22(10-40.49)$ \\
\hline Blood urea nitrogen (mg/dL) & 2 & $7-18$ & $48.1(40.7-55.5)$ \\
\hline \multicolumn{4}{|l|}{ Coagulation } \\
\hline D-dimer (mg/L) & 10 & $<0.4$ & $3.2(0.29-619)$ \\
\hline Fibrinogen (mg/dL) & 3 & $220-440$ & $418(364.74-730)$ \\
\hline Erythrocyte sedimentation rate $(\mathrm{mm} / \mathrm{h})$ & 2 & $0-20$ & $29(13-45)$ \\
\hline
\end{tabular}

Lastly, Table 5 presented a comparison of CSF analysis between the CNS group and the PNS group. Patients with CNS disorders were younger (2.785 years old; 0-16 years old) compared to those with PNS manifestations (12.5 years old; 4-15 years old). The median for the onset of lumbar puncture from the first symptom was two days (0-18 days) in the CNS group, while it took three days (1-10 days) in the PNS group. None of the PNS group tested positive for COVID-19 from the CSF sample, while five pediatric patients were positive for COVID-19 via CSF analysis. In the CNS group, CSF monocytes (55\%; $11-80 \%$ ), CSF polymorphonuclear cells (15\%; 0-96\%), CSF red blood cells (282 cells $/ \mathrm{mm}^{3} ; 2-4000$ cells $\left./ \mathrm{mm}^{3}\right)$, and opening pressure $\left(34.5 \mathrm{~cm} / \mathrm{H}_{2} \mathrm{O} ; 25-100 \mathrm{~cm} / \mathrm{H}_{2} \mathrm{O}\right)$ were elevated. Meanwhile, in the PNS group, all the parameters were mostly normal except for positive albumin-cytological dissociation. 
Table 5

Cerebrospinal fluid analysis according to central or peripheral nervous system involvement

\begin{tabular}{|c|c|c|c|}
\hline Variable & $\begin{array}{l}\text { Reference } \\
\text { range }\end{array}$ & $\begin{array}{l}\text { Central nervous system disorder }(n) \text {; } \\
\text { median (range)* }\end{array}$ & $\begin{array}{l}\text { Peripheral nervous system disorder }(\mathrm{n}) \text {; } \\
\text { median (range)* }\end{array}$ \\
\hline Total & $\mathrm{N} / \mathrm{A}$ & 38 & 4 \\
\hline Age & $\mathrm{N} / \mathrm{A}$ & $2.785(0-16)$ & $12.5(4-15)$ \\
\hline Male sex & $\mathrm{N} / \mathrm{A}$ & 23 & 2 \\
\hline $\begin{array}{l}\text { Onset from first symptom to lumbar } \\
\text { puncutre (days) }\end{array}$ & N/A & $2(0-18) ; n=21$ & $3(1-10)$ \\
\hline CSF PCR results for COVID-19 & Negative & Positive: 5; Negative: 12; Not checked: 21 & Negative: 1 ; Not checked: 3 \\
\hline Total cells (count/ $\mu \mathrm{L})$ & $0-20$ & $6.5(0-55) ; n=16$ & $5(5-5) ; n=2$ \\
\hline Normal value (n) & & $20 ; n=23$ & 4 \\
\hline Increased (n) & & $3 ; n=23$ & 0 \\
\hline CSF lymphocytes (\%) & $40-80$ & $41.5(20-89) ; n=4$ & $91 ; n=1$ \\
\hline Normal value (n) & & $3 ; n=6$ & 3 \\
\hline Increased (n) & & $1 ; n=6$ & 1 \\
\hline Decreased (n) & & $2 ; n=6$ & 0 \\
\hline CSF monocytes (\%) & $15-45$ & $55(11-80) ; n=3$ & $9 ; n=1$ \\
\hline Normal value (n) & & $3 ; n=6$ & 3 \\
\hline Increased (n) & & $2 ; n=6$ & 0 \\
\hline Decreased (n) & & $1 ; n=6$ & 1 \\
\hline CSF polymorphonuclear cells (\%) & $0-6 \%$ & $15(0-96) ; n=9$ & N/A \\
\hline Normal value (n) & & $10 ; n=16$ & $2 ; n=2$ \\
\hline Increased (n) & & $5 ; n=16$ & $0 ; n=2$ \\
\hline Decreased (n) & & $1 ; n=16$ & $0 ; n=2$ \\
\hline CSF protein (mg/dL) & $5-40$ & $40(0.3-97) ; n=13$ & 34.7 (25-316.7); n = 3 \\
\hline Normal value (n) & & $16 ; n=22$ & 2 \\
\hline Increased (n) & & $5 ; n=22$ & 1 \\
\hline Decreased (n) & & $1 ; n=22$ & 0 \\
\hline CSF glucose (mg/dL) & $40-80$ & $60(3.1-92) ; n=13$ & $65(64-66), n=3$ \\
\hline Normal value (n) & & $18 ; n=22$ & 4 \\
\hline Increased (n) & & $3 ; n=22$ & 0 \\
\hline Decreased (n) & & $1 ; n=22$ & 0 \\
\hline CSF red blood cells $\left(\mathrm{RBC} / \mathrm{mm}^{3}\right)$ & 0 & $282(2-4000) ; n=9$ & N/A \\
\hline Normal value (n) & & $7 ; n=16$ & $2 ; n=2$ \\
\hline Increased (n) & & $9 ; n=16$ & $0 ; n=2$ \\
\hline Decreased (n) & & $0 ; n=16$ & $0 ; n=2$ \\
\hline Opening pressure $\left(\mathrm{cm} / \mathrm{H}_{2} \mathrm{O}\right)$ & $11.5-28$ & $34.5(25-100) ; n=4$ & N/A \\
\hline Normal value (n) & & $4 ; n=7$ & $1 ; n=1$ \\
\hline Increased (n) & & $3 ; n=7$ & $0 ; n=1$ \\
\hline Decreased (n) & & $0 ; n=7$ & $0 ; n=1$ \\
\hline Positive oligoclonal bands & Negative & $0 ; n=3$ & $0 ; n=1$ \\
\hline \multicolumn{4}{|c|}{$\begin{array}{l}\text { The reference range used in this table is according to normal values of children who are } 3.5 \text { years old (the median age of this study). These values are } \\
\text { obtained from The Harriet Lane Handbook (78) and Mosby's Manual of Diagnostic and Laboratory Tests (79). }\end{array}$} \\
\hline \multicolumn{4}{|c|}{ *When $\mathrm{n}$ is not defined, $\mathrm{n}$ has the same value as the total sample } \\
\hline CSF, cerebrospinal fluid; PCR, polyme & n reaction; & red blood cells; N/A, not available & \\
\hline
\end{tabular}




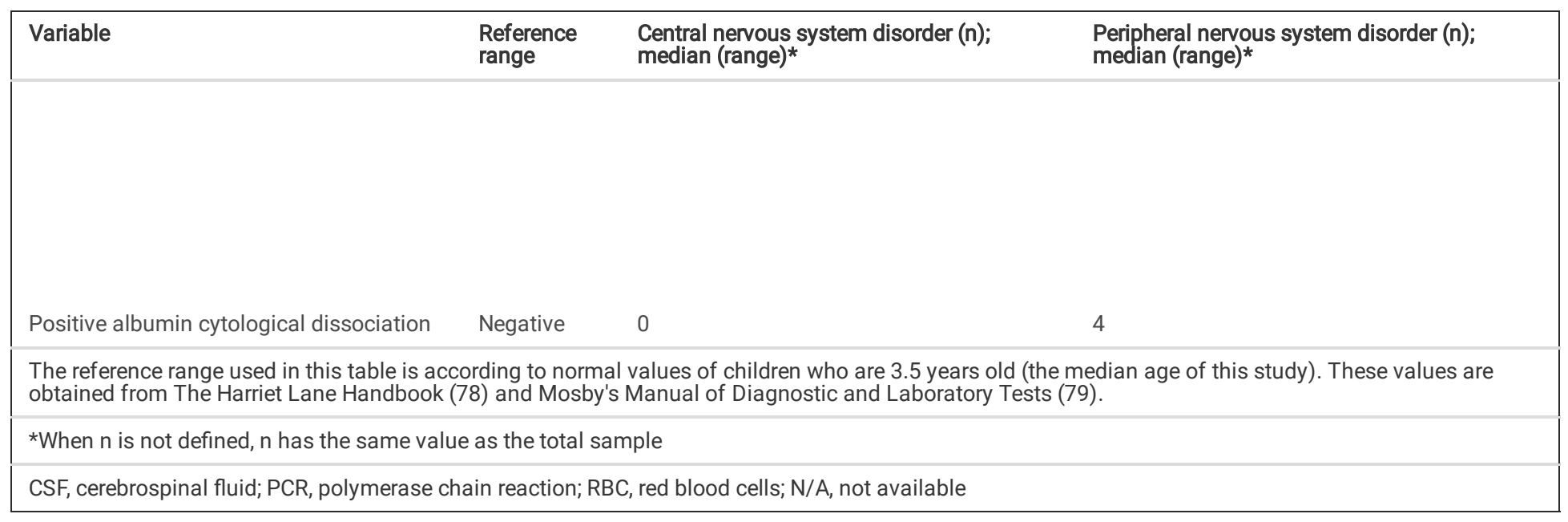

\section{Discussion}

Our systematic review only includes 31 studies with 42 patients, a far cry from another systematic review that has been done with 26 studies and 3707 patients (19). However, Panda et al. only focus on reporting the neurological symptoms while this systematic review requires investigations of neurological symptoms, explaining the wide gap in the number of patients (19). Other systematic reviews that have also been done primarily focus on neurological manifestations in adults $(14,15,17)$.

Encephalopathy is the most prominent and definite neurological complication, which some of the cases might be mediated by hypoxia and septic shock (19). This finding is similar to our result, where encephalomyelopathy and seizure are among the most common neurological diagnoses in COVID-19. The exact pathomechanism of SARS-CoV-2 infections on the nervous system has not been fully elucidated yet. Proposed mechanisms are direct infection injury via the hematogenous and neuronal pathway, hypoxia injury, immune injury, and angiotensin-converting enzyme-2 (ACE-2) (16). A high ACE-2 expression is found in the brainstem, cerebral cortex, amygdala, with the highest ACE-2 expression level at pons and medulla oblongata. The brainstem contains medullary respiratory centers, and this may explain the susceptibility of COVID-19 patients to respiratory distress (35).

Acute ischemic stroke is the most common diagnosis in this systematic review. Studies have shown an increase in the incidence of ischemic stroke by $2.5 \%$ in Italy and 3.7\% in the Netherlands (36). This represents the cerebrovascular system's thromboembolic mechanism, found in COVID-19 patients, albeit more common in adult patients (18). Other possible pathways include the ACE-2 receptor and its subsequent effect on blood flow, hypoxia, vascular endothelial injury, and inflammatory insults $(13,20)$. The high cases of reported acute ischemic stroke in pediatric patients may reflect increased awareness of neurological complications of pediatric COVID-19 patients as acute ischemic stroke is uncommon in adult COVID-19 cases with a pooled prevalence of 2.1\% $(14,17)$.

Seizure and altered mental consciousness are the two most common neurological manifestations. While seizure is not the most predominant symptom in other studies, it is more common in patients with severe COVID-19 disease, especially in multisystem inflammatory responses (37). This is why the authors excluded MIS-C in this systematic review because neurological manifestations are higher in MIS-C, which might skew the results (18, 20). Interestingly, most of the patients in this systematic review present with moderate severity, which might imply ongoing and evolving inflammation, leading to clinical deterioration. Hence, clinicans should keep a close observation of pediatric patients with neurological manifestations (38).

Headache is the most common CNS symptom, and it is regarded as secondary to hypoxia $(13,14,17,19)$. However, the most common PNS symptom is anosmia and ageusia $(13,14,17)$, which is not found in our result. Inflammation of the olfactory nerves might explain anosmia, while ACE-2 receptor damage on the tongue might explain ageusia. It is noteworthy that these studies include adults as their population, whereas it is more difficult to extract this information in children, especially those with severe symptoms (19). Qiu et al. look at the characteristics of adults and children presenting with olfactory or gustatory dysfunction. They find that $37 \%$ of the children present with olfactory or gustatory dysfunction with a median age of 16.6 years old, which shows that adolescents are more aware and more likely to report such symptoms than children (39). This study is not included in our systematic review because no further investigations are done on the patients.

Most of the patients are alive except for three patients. All three deaths can be explained by critical presentation, complications of surgery while, and cardiopulmonary arrest (40-42). Increased C-reactive protein, D-dimer levels, creatinine kinase, ferritin, procalcitonin, erythrocyte sedimentation rate, and decreased lymphocytes may portend poorer prognosis and hence leading to increased severity of disease and mortality $(14,43,44)$.

One systematic review found that most patients who present with PNS manifestation have a normal cell count and high protein (22). This is different from our studies, where all CSF parameters are within normal except for positive albumin-cytological dissociation. Some of the possible explanations for this finding are that more samples in the PNS group $(n=55)$ and adult population are used in that systematic review. A different time course in obtaining the CSF sample may alter the CSF results, which may contribute to different results found in our study (45). Espíndola et al. found that there is pleocytosis with predominant mononuclear cells in CNS disorders, a mild increase in total protein concentration and high neurofilament light (21), which is similar to what we find. There are only five samples that test positive for COVID-19 from CSF samples. This may be due to the low sensitivity of SARS-CoV-2 detection using PCR, the onset of 
manifestation to lumbar puncture, as well as different pathomechanisms that may alter the results $(19,20)$. Therefore, any negative PCR results from CSF need to be interpreted with caution (46).

There are several limitations to our study. Firstly, many studies are excluded due to not meeting the criteria of investigations of neurological symptoms, or the corresponding authors do not provide essential data for this systematic review $(23,25,26,47-52)$. Despite many studies not being included in this systematic review, there is no guarantee that all those studies will end up included due to inclusion and exclusion criteria imposed. Secondly, our systematic review has a low sample, with most of the studies being case reports. This reflects the rarity of pediatric neurologic COVID-19 infections investigated by either laboratory or imaging studies. This systematic review gives insights into the clinical profiles and CSF analysis in pediatric COVID-19 patients with neurologic symptoms despite the low samples. Lastly, not all the data required in this systematic review are given in the corresponding paper. To our knowledge, this is the first systematic review to elucidate CSF analysis from pediatric COVID-19 patients with neurological symptoms.

\section{Conclusion}

This systematic review describes the demographic status, clinical manifestations, laboratory and imaging studies, including CSF analysis of new-onset and investigated pediatric COVID-19 patients. There is a male predominance with a majority of CNS diagnoses as well as a good prognosis. Results of CSF analysis show that CNS disorders present with elevated CSF monocytes, CSF polymorphonuclear cells, CSF red blood cells, and opening pressure. In contrast, patients with PNS disorders mostly have normal values except for albumin-cytological dissociation. Further studies are required to find the long-term consequences of neurological complications of COVID-19 as well as the dynamics of CSF results in pediatric COVID-19 patients.

\section{Declarations}

\section{Conflicts of interests}

The authors declare that they have no known competing financial interests or personal relationships that could have influenced the work reported in this paper.

\section{Funding}

Any institutions do not fund this study.

\section{Acknowledgment}

We would like to thank Dr. Dragan Savić and Dr. Thirugnanam Umapathi for providing us with additional data or confirmation about their respective study to complete this systematic review.

\section{Availability of data and material}

Data will be given upon reasonable request

\section{Ethics approval}

Not available

\section{Consent to participate}

Not available

\section{Consent for publication}

All authors have read and agreed on publishing this article

\section{Authors' contributions}

GSO and AJ did the conception of this systematic review while GSO registered the procedure on PROSPERO website. Data collections are done by GSO, SC, and JHW with disputes between the articles that were selected settled by AJ. GSO, SC, and JHW drafted the article while AJ did critical revision of the article. Final approval of the version to be published was granted by all authors.

\section{References}

1. Gostin LO. The Coronavirus Pandemic 1 Year On-What Went Wrong? JAMA Health Forum. 2021;2(2):e210174-e.

2. Mishra SK, Tripathi T. One year update on the COVID-19 pandemic: Where are we now? Acta Tropica. 2021;214:105778.

3. Güner R, Hasanoğlu I, Aktaş F. COVID-19: Prevention and control measures in community. Turk J Med Sci. 2020;50(SI-1):571-7.

4. Moore JP. Approaches for Optimal Use of Different COVID-19 Vaccines: Issues of Viral Variants and Vaccine Efficacy. Jama. 2021;325(13):1251-2.

5. Olliaro P, Torreele E, Vaillant M. COVID-19 vaccine efficacy and effectiveness-the elephant (not) in the room. The Lancet Microbe. 2021.

6. Kelly K, Hwei LRY, Octavius GS. Coronavirus outbreaks including COVID-19 and impacts on medical education: a systematic review. Journal of Community Empowerment for Health. 2020;3(2):130-40. 
7. Haug N, Geyrhofer L, Londei A, Dervic E, Desvars-Larrive A, Loreto V, et al. Ranking the effectiveness of worldwide COVID-19 government interventions. Nature Human Behaviour. 2020;4(12):1303-12.

8. Jain U. Effect of COVID-19 on the Organs. Cureus. 2020;12(8):e9540-e.

9. Octavius GS, Silviani FR, Lesmandjaja A, Angelina, Juliansen A. Impact of COVID-19 on adolescents' mental health: a systematic review. Middle East Current Psychiatry. 2020;27(1):72.

10. Desai I, Manchanda R, Kumar N, Tiwari A, Kumar M. Neurological manifestations of coronavirus disease 2019: exploring past to understand present. Neurol Sci. 2021;42(3):773-85.

11. Rahman A, Niloofa R, De Zoysa IM, Cooray AD, Kariyawasam J, Seneviratne SL. Neurological manifestations in COVID-19: A narrative review. SAGE Open Medicine. 2020;8:2050312120957925.

12. Ahmad I, Rathore FA. Neurological manifestations and complications of COVID-19: A literature review. Journal of Clinical Neuroscience. 2020;77:8-12.

13. Padda I, Khehra N, Jaferi U, Parmar MS. The Neurological Complexities and Prognosis of COVID-19. SN Compr Clin Med. 2020:1-12.

14. Favas TT, Dev P, Chaurasia RN, Chakravarty K, Mishra R, Joshi D, et al. Neurological manifestations of COVID-19: a systematic review and meta-analysis of proportions. Neurological Sciences. 2020;41(12):3437-70.

15. Asadi-Pooya AA, Simani L. Central nervous system manifestations of COVID-19: A systematic review. Journal of the neurological sciences. 2020;413:116832.

16. Wu Y, Xu X, Chen Z, Duan J, Hashimoto K, Yang L, et al. Nervous system involvement after infection with COVID-19 and other coronaviruses. Brain, behavior, and immunity. 2020;87:18-22.

17. Nepal G, Rehrig JH, Shrestha GS, Shing YK, Yadav JK, Ojha R, et al. Neurological manifestations of COVID-19: a systematic review. Critical care (London, England). 2020;24(1):421.

18. Chen TH. Neurological involvement associated with COVID-19 infection in children. Journal of the neurological sciences. 2020;418:117096.

19. Panda PK, Sharawat IK, Panda P, Natarajan V, Bhakat R, Dawman L. Neurological Complications of SARS-CoV-2 Infection in Children: A Systematic Review and Meta-Analysis. Journal of tropical pediatrics. 2020.

20. Lin JE, Asfour A, Sewell TB, Hooe B, Pryce P, Earley C, et al. Neurological issues in children with COVID-19. Neuroscience letters. 2021;743:135567.

21. Espíndola OdM, Siqueira M, Soares CN, Lima MASDd, Leite ACCB, Araujo AQC, et al. Patients with COVID-19 and neurological manifestations show undetectable SARS-CoV-2 RNA levels in the cerebrospinal fluid. International Journal of Infectious Diseases. 2020;96:567-9.

22. Tandon M, Kataria S, Patel J, Mehta TR, Daimee M, Patel V, et al. A Comprehensive Systematic Review of CSF analysis that defines Neurological Manifestations of COVID-19. International journal of infectious diseases : IJID : official publication of the International Society for Infectious Diseases. 2021;104:390-7.

23. Destras G, Bal A, Escuret V, Morfin F, Lina B, Josset L. Systematic SARS-CoV-2 screening in cerebrospinal fluid during the COVID-19 pandemic. The Lancet Microbe. 2020;1(4):e149.

24. Bai K, Liu W, Liu C, Fu Y, Hu J, Qin Y, et al. Clinical Analysis of 25 COVID-19 Infections in Children. The Pediatric infectious disease journal. 2020;39(7):e100-e3.

25. Kremer S, Lersy F, de Sèze J, Ferré JC, Maamar A, Carsin-Nicol B, et al. Brain MRI Findings in Severe CoVID-19: A Retrospective Observational Study. Radiology. 2020;297(2):E242-e51.

26. Garazzino S, Montagnani C, Donà D, Meini A, Felici E, Vergine G, et al. Multicentre Italian study of SARS-CoV-2 infection in children and adolescents, preliminary data as at 10 April 2020. Euro surveillance : bulletin Europeen sur les maladies transmissibles = European communicable disease bulletin . 2020;25(18).

27. Page MJ, McKenzie JE, Bossuyt PM, Boutron I, Hoffmann TC, Mulrow CD, et al. The PRISMA 2020 statement: an updated guideline for reporting systematic reviews. Systematic Reviews. 2021;10(1):89.

28. Dong Y, Mo X, Hu Y, Qi X, Jiang F, Jiang Z, et al. Epidemiology of COVID-19 Among Children in China. Pediatrics. 2020;145(6).

29. Joanna Briggs I. The Joanna Briggs Institute Critical Appraisal tools for use in JBI Systematic Reviews: Checklists for Case Reports: The Joanna Briggs Institute; 2019 [cited $202117^{\text {th }}$ April]. Available from: https://jbi.global/sites/default/files/2019-05/JBI_Critical_Appraisal-

Checklist_for_Case_Reports2017_0.pdf.

30. Wells G, Shea B, O'Connell D, Peterson J, Welch, Losos M, et al., editors. The Newcastle-Ottawa Scale (NOS) for Assessing the Quality of Nonrandomised Studies in Meta-Analyses2014.

31. Miller EH, Namale VS, Kim C, Dugue R, Waldrop G, Ciryam P, et al. Cerebrospinal Analysis in Patients With COVID-19. Open forum infectious diseases. 2020;7(11):ofaa501.

32. Paterson RW, Brown RL, Benjamin L, Nortley R, Wiethoff S, Bharucha T, et al. The emerging spectrum of COVID-19 neurology: clinical, radiological and laboratory findings. Brain : a journal of neurology. 2020;143(10):3104-20.

33. Emami A, Fadakar N, Akbari A, Lotfi M, Farazdaghi M, Javanmardi F, et al. Seizure in patients with COVID-19. Neurol Sci. 2020;41(11):3057-61.

34. Beslow LA, Linds AB, Fox CK, Kossorotoff M, Zuñiga Zambrano YC, Hernández-Chávez M, et al. Pediatric Ischemic Stroke: An Infrequent Complication of SARS-CoV-2. Annals of neurology. 2020.

35. Lukiw WJ, Pogue A, Hill JM. SARS-CoV-2 Infectivity and Neurological Targets in the Brain. Cellular and Molecular Neurobiology. 2020.

36. Bridwell R, Long B, Gottlieb M. Neurologic complications of COVID-19. Am J Emerg Med. 2020;38(7):1549.e3-.e7. 
37. Correia AO, Feitosa PWG, Moreira JLdS, Nogueira SÁR, Fonseca RB, Nobre MEP. Neurological manifestations of COVID-19 and other coronaviruses: A systematic review. Neurol Psychiatry Brain Res. 2020;37:27-32.

38. Ish P, Sachdev K, Agrawal S, Gupta N. Neurological Manifestations of COVID-19 - Continually Evolving and Perplexing. Ann Indian Acad Neurol. 2020;23(3):361-2.

39. Qiu C, Cui C, Hautefort C, Haehner A, Zhao J, Yao Q, et al. Olfactory and Gustatory Dysfunction as an Early Identifier of COVID-19 in Adults and Children: An International Multicenter Study. Otolaryngology-Head and Neck Surgery. 2020;163(4):714-21.

40. Conto-Palomino NM, Cabrera-Bueno ML, Vargas-Ponce KG, Rondón-Abuhadba EA, Atamari-Anahui N. Encephalitis associated with COVID-19 in a 13-yearold girl: A case report. Medwave. 2020;20(7):e7984.

41. Gutierrez Amezcua JM, Jain R, Kleinman G, Muh CR, Guzzetta M, Folkerth R, et al. COVID-19-Induced Neurovascular Injury: a Case Series with Emphasis on Pathophysiological Mechanisms. SN Compr Clin Med. 2020;2(11):2109-25.

42. Savić D, Alsheikh TM, Alhaj AK, Lazovic L, Alsarraf L, Bosnjakovic P, et al. Ruptured cerebral pseudoaneurysm in an adolescent as an early onset of COVID-19 infection: case report. Acta Neurochir (Wien). 2020;162(11):2725-9.

43. Elshazli RM, Toraih EA, Elgaml A, El-Mowafy M, El-Mesery M, Amin MN, et al. Diagnostic and prognostic value of hematological and immunological markers in COVID-19 infection: A meta-analysis of 6320 patients. PLOS ONE. 2020;15(8):e0238160.

44. Pourbagheri-Sigaroodi A, Bashash D, Fateh F, Abolghasemi H. Laboratory findings in COVID-19 diagnosis and prognosis. Clinica chimica acta; international journal of clinical chemistry. 2020;510:475-82.

45. Illes Z, Blaabjerg M. Cerebrospinal fluid findings in Guillain-Barré syndrome and chronic inflammatory demyelinating polyneuropathies. Handbook of clinical neurology. 2017;146:125-38.

46. Poyiadji N, Shahin G, Noujaim D, Stone M, Patel S, Griffith B. COVID-19-associated Acute Hemorrhagic Necrotizing Encephalopathy: Imaging Features. Radiology. 2020;296(2):E119-E20.

47. Qiu H, Wu J, Hong L, Luo Y, Song Q, Chen D. Clinical and epidemiological features of 36 children with coronavirus disease 2019 (COVID-19) in Zhejiang, China: an observational cohort study. The Lancet Infectious Diseases. 2020;20(6):689-96.

48. Li Y, Wang H, Wang F, Du H, Liu X, Chen P, et al. Comparison of hospitalized patients with pneumonia caused by COVID-19 and influenza A in children under 5 years. International journal of infectious diseases : IJID : official publication of the International Society for Infectious Diseases. 2020;98:80-3.

49. Zhang B, Liu S, Zhang J, Xiao J, Zhu S, Dong Y, et al. Children hospitalized for coronavirus disease 2019 (COVID-19): A multicenter retrospective descriptive study. J Infect. 2020;81(2):e74-e5.

50. Nathan N, Prevost B, Sileo C, Richard N, Berdah L, Thouvenin G, et al. The Wide Spectrum of COVID-19 Clinical Presentation in Children. Journal of clinical medicine. 2020;9(9).

51. Wu H, Zhu H, Yuan C, Yao C, Luo W, Shen X, et al. Clinical and Immune Features of Hospitalized Pediatric Patients With Coronavirus Disease 2019 (COVID19) in Wuhan, China. JAMA Network Open. 2020;3(6):e2010895-e.

52. Lindan CE, Mankad K, Ram D, Kociolek LK, Silvera VM, Boddaert N, et al. Neuroimaging manifestations in children with SARS-CoV-2 infection: a multinational, multicentre collaborative study. The Lancet Child \& Adolescent Health. 2021;5(3):167-77.

53. Nathan N, Prevost B, Corvol H. Atypical presentation of COVID-19 in young infants. Lancet (London, England). 2020;395(10235):1481-.

54. McAbee GN, Brosgol Y, Pavlakis S, Agha R, Gaffoor M. Encephalitis Associated with COVID-19 Infection in an 11-Year-Old Child. Pediatr Neurol. 2020;109:94-

55. Burr T, Barton C, Doll E, Lakhotia A, Sweeney M. N-Methyl-d-Aspartate Receptor Encephalitis Associated With COVID-19 Infection in a Toddler. Pediatr Neurol. 2021;114:75-6.

56. Chacón-Aguilar R, Osorio-Cámara JM, Sanjurjo-Jimenez I, González-González C, López-Carnero J, Pérez-Moneo B. COVID-19: Fever syndrome and neurological symptoms in a neonate. An Pediatr (Engl Ed). 2020;92(6):373-4.

57. Di Nicola P, Ceratto S, Dalmazzo C, Roasio L, Castagnola E, Sannia A. Concomitant SARS-CoV-2 infection and severe neurologic involvement in a latepreterm neonate. Neurology. 2020;95(18):834-5.

58. Chegondi M, Kothari H, Chacham S, Badheka A. Coronavirus Disease 2019 (COVID-19) Associated With Febrile Status Epilepticus in a Child. Cureus. 2020;12(8):e9840-e.

59. Paybast S, Gorji R, Mavandadi S. Guillain-Barré Syndrome as a Neurological Complication of Novel CoVID-19 Infection: A Case Report and Review of the Literature. The neurologist. 2020;25(4):101-3.

60. Khalifa M, Zakaria F, Ragab Y, Saad A, Bamaga A, Emad Y, et al. Guillain-Barré Syndrome Associated With Severe Acute Respiratory Syndrome Coronavirus 2 Detection and Coronavirus Disease 2019 in a Child. Journal of the Pediatric Infectious Diseases Society. 2020;9(4):510-3.

61. Frank CHM, Almeida TVR, Marques EA, de Sousa Monteiro Q, Feitoza PVS, Borba MGS, et al. Guillain-Barré Syndrome Associated with SARS-CoV-2 Infection in a Pediatric Patient. Journal of tropical pediatrics. 2020;0:1-6.

62. Shen MY, Dugue R, Maldonado-Soto AR, Thakur KT, Zyskind I, Vargas WS. Acute Ischemic Stroke in a Pediatric Patient With Known Exposure to COVID-19 and Positive Serology. Pediatr Neurol. 2021;116:39-40.

63. de Ruijter NS, Kramer G, Gons RAR, Hengstman GJD. Neuromyelitis optica spectrum disorder after presumed coronavirus (COVID-19) infection: A case report. Multiple sclerosis and related disorders. 2020;46:102474.

64. Dugue R, Cay-Martínez KC, Thakur KT, Garcia JA, Chauhan LV, Williams SH, et al. Neurologic manifestations in an infant with COVID-19. Neurology. 2020;94(24):1100-2. 
65. Khan A, Panwala H, Ramadoss D, Khubchandani R. Myelin Oligodendrocyte Glycoprotein (MOG) Antibody Disease in a 11 Year Old with COVID-19 Infection. Indian journal of pediatrics. 2021:1-2.

66. Dominguez J, Estupiñan M, Garcés-Ghilardi R, Alvarado-Gamarra G, Stapleton A, Seminario R, et al. Extra pulmonary COVID-19 manifestations in pediatrics: report of three cases Hospital Edgardo Rebagliati Martins - Peru. Scielo Preprints. 2020:1-9.

67. Seth V, Kushwaha S. Headache Due to COVID-19: A Disabling Combination. Headache. 2020;60(10):2618-21.

68. Mirzaee SMM, Gonçalves FG, Mohammadifard M, Tavakoli SM, Vossough A. Focal Cerebral Arteriopathy in a Pediatric Patient with COVID-19. Radiology. 2020;297(2):E274-e5.

69. Kaur H, Mason JA, Bajracharya M, McGee J, Gunderson MD, Hart BL, et al. Transverse Myelitis in a Child With COVID-19. Pediatr Neurol. 2020;112:5-6.

70. Bhatta S, Sayed A, Ranabhat B, Bhatta RK, Acharya Y. New-Onset Seizure as the Only Presentation in a Child With COVID-19. Cureus. 2020;12(6):e8820-e.

71. Swarz JA, Daily S, Niemi E, Hilbert SG, Ibrahim HA, Gaitanis JN. COVID-19 Infection Presenting as Acute-Onset Focal Status Epilepticus. Pediatr Neurol. 2020;112:7.

72. Davide S, Pietro S, Francesca D, Paolo B. Self-limited focal epilepsy in a young child with SARS-CoV-2: serendipity or causal association? Research Square. 2021; Preprint (Version 1).

73. Vivanti AJ, Vauloup-Fellous C, Prevot S, Zupan V, Suffee C, Do Cao J, et al. Transplacental transmission of SARS-CoV-2 infection. Nature communications. 2020;11(1):3572.

74. Gulko E, Overby P, Ali S, Mehta H, Al-Mufti F, Gomes W. Vessel Wall Enhancement and Focal Cerebral Arteriopathy in a Pediatric Patient with Acute Infarct and COVID-19 Infection. AJNR American journal of neuroradiology. 2020;41(12):2348-50.

75. Lorenz N, Treptow A, Schmidt S, Hofmann R, Raumer-Engler M, Heubner G, et al. Neonatal Early-Onset Infection With SARS-CoV-2 in a Newborn Presenting With Encephalitic Symptoms. The Pediatric infectious disease journal. 2020;39(8):e212.

76. Tan YP, Tan BY, Pan J, Wu J, Zeng SZ, Wei HY. Epidemiologic and clinical characteristics of 10 children with coronavirus disease 2019 in Changsha, China. J Clin Virol. 2020;127:104353.

77. Pesce M. Reference Intervals for Laboratory Tests and Procedures. In: Kliegman R, Behrman R, Jenson H, Stanton B, editors. Nelson Textbook of Pediatrics. 18 ed. Philadelphia: WB Saunders; 2011. p. 2943-9.

78. Hughes H, Kahl L. Blood Chemistries and Body Fluids. In: Hughes H, Kahl L, editors. The Harriet Lane handbook : a manual for pediatric house officers / The Harriet Lane Service at The Charlotte R Bloomberg Children's Center of The Johns Hopkins Hospital. 21 ed. Philadelphia: Elsevier; 2018. p. 717.

79. Pagana K, Pagana T. Mosby's Manual of Diagnostic and Laboratory Tests. 5 ed. Missouri: Elsevier; 2014. 651-2 p.

\section{Figures}

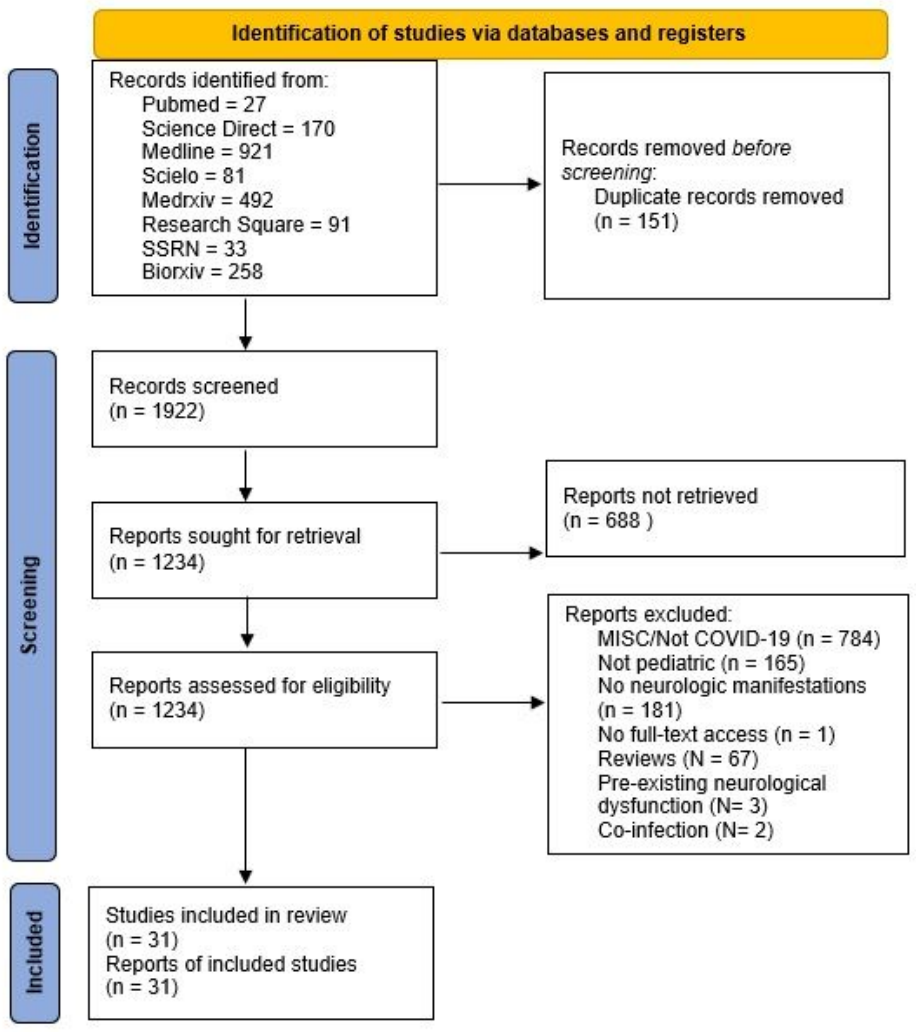

Figure 1

Page $17 / 19$ 
PRISMA flow chart of this study

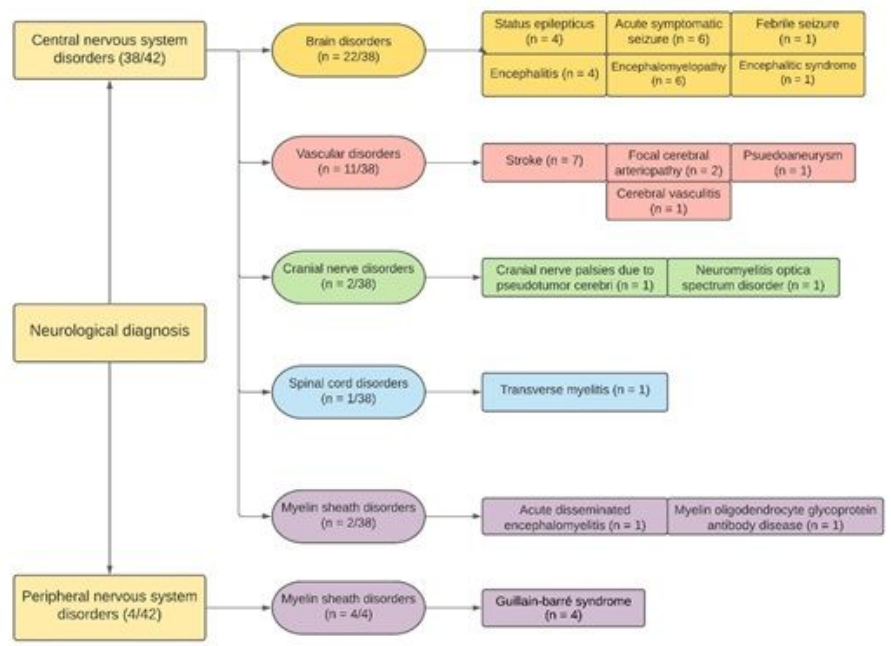

Figure 2

Classification of COVID-19 induced neurological disorders

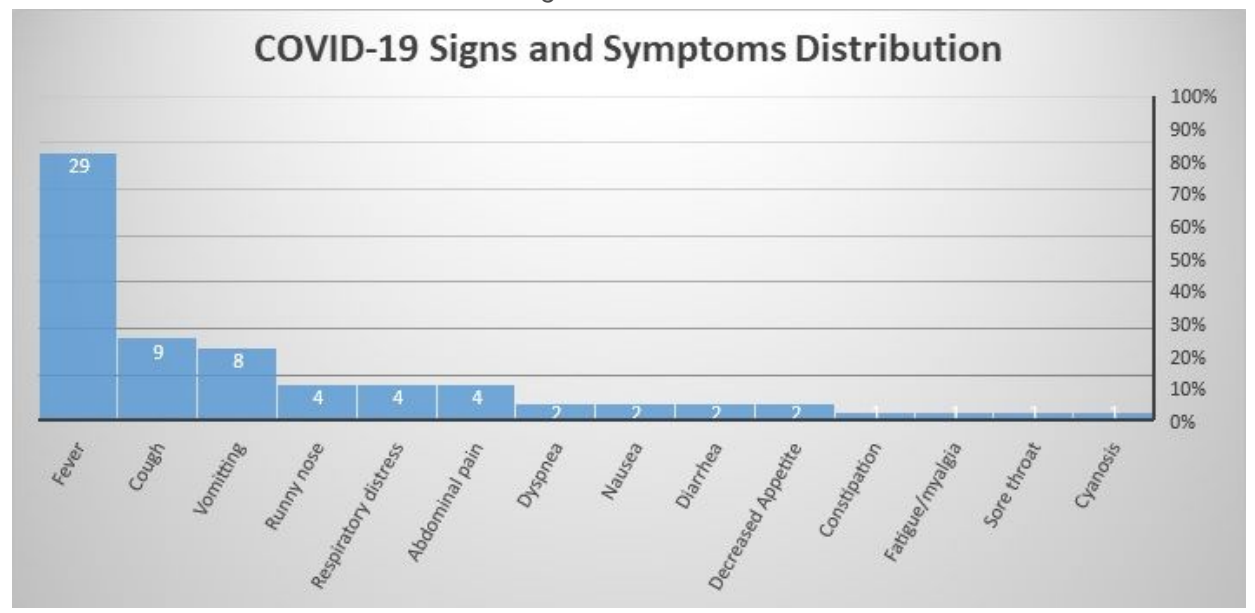

Figure 3

COVID-19 signs and symptoms distribution

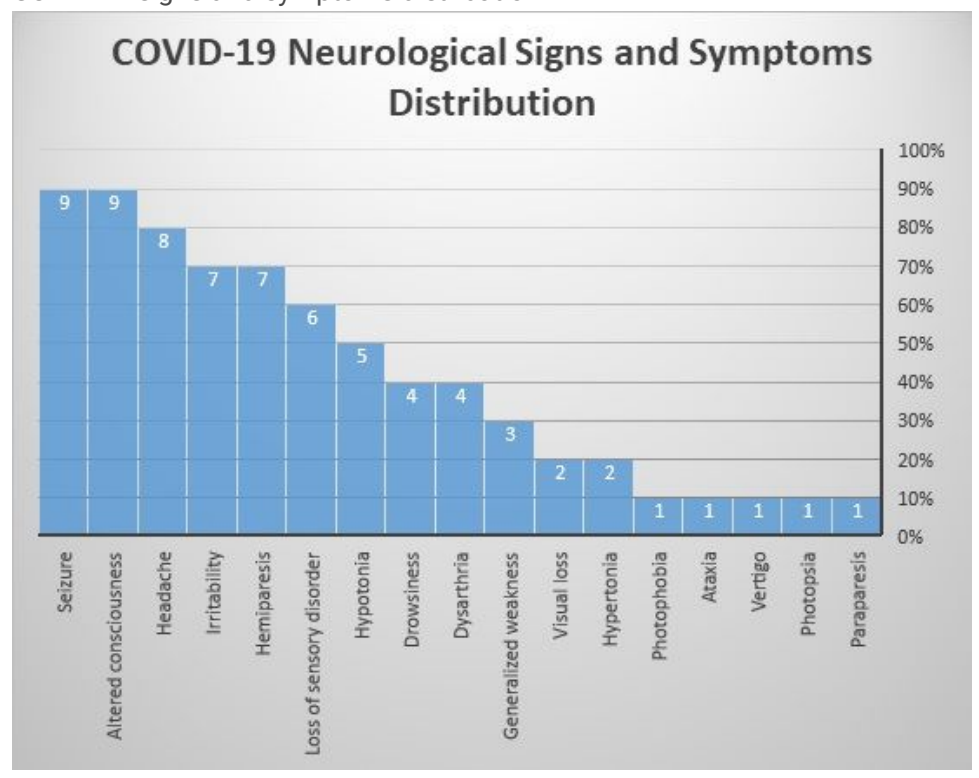

Figure 4 
COVID-19 neurological signs and symptoms distribution

\section{Supplementary Files}

This is a list of supplementary files associated with this preprint. Click to download.

- coidisclosureAJ.pdf

- coidisclosureGilbert.pdf

- coidisclosureJeremy.pdf

- coidisclosureShally.pdf 